\title{
A Dynamical Approach to Accelerating Numerical Integration with Equidistributed Points
}

\author{
O. Jenkinson ${ }^{a}$ and M. Pollicott ${ }^{b}$
}

Received October 2006

\begin{abstract}
We show how ideas originating in the theory of dynamical systems inspire a new approach to numerical integration of functions. Any Lebesgue integral can be approximated by a sequence of integrals with respect to equidistributions, i.e. evenly weighted discrete probability measures concentrated on an equidistributed set. We prove that, in the case where the integrand is real analytic, suitable linear combinations of these equidistributions lead to a significant acceleration in the rate of convergence of the approximate integral. In particular, the rate of convergence is faster than that of any Newton-Cotes rule.
\end{abstract}

DOI: $10.1134 / \mathrm{S} 0081543807010166$

\section{INTRODUCTION}

Numerical integration involves approximating an integral over a continuous space by a discrete summation over a finite set of points, for example,

$$
\int_{0}^{1} f(x) d x \approx \frac{1}{n} \sum_{k=1}^{n} f\left(\frac{k}{n}\right) .
$$

An improved method of numerically approximating $\int f(x) d x$ is to form a weighted sum of the same values $f(j / n)$. For example, the well-known Simpson's rule allows us to write

$$
\int_{0}^{1} f(x) d x \approx \frac{1}{6 n}\left(\sum_{k=1}^{n} 4 f\left(\frac{k}{2 n}\right)+2 \sum_{k=1}^{n} f\left(\frac{2 k-1}{2 n}\right)\right)
$$

with, typically, a smaller error. Both (1) and (2) are special cases of (composite) Newton-Cotes integral rules (see, e.g., [13]). Higher order Newton-Cotes integral rules are derived from interpolation of higher order polynomials, and again involve weighted sums of evaluations of $f$ at the points in the equidistributed set

$$
\mathcal{P}_{n}=\left\{\frac{j}{n}: j \in\{0,1, \ldots, n\}\right\}
$$

For these classical methods of numerical integration, the error term (i.e., the difference between the actual and approximate integrals) is $O\left(n^{-l}\right)$ as $n \rightarrow \infty$ (see, e.g., [13]) for some $l>0$.

Although smaller error terms can be obtained by evaluating $f$ on sets which are not equidistributed (e.g., as in Gaussian quadrature, cf. [13]), the question we shall address here is as follows: Is there a more efficient method for approximating integrals based on integrand evaluation at equidistributed points?

\footnotetext{
${ }^{a}$ School of Mathematical Sciences, Queen Mary, University of London, Mile End Road, London, E1 4NS, UK.

${ }^{b}$ Mathematics Institute, University of Warwick, Coventry, CV4 7AL, UK.

E-mail addresses: omj@maths.qmul.ac.uk (O. Jenkinson), mpollic@maths.warwick.ac.uk (M. Pollicott).
} 
Our main conclusion (see Theorems 1 and 2) is that if $f:[0,1] \rightarrow \mathbb{R}$ is real analytic, then the convergence can be considerably accelerated.

For any $M \in \mathbb{N}$, define the equidistribution

$$
E_{M}=\frac{1}{M+1} \sum_{j=0}^{M} \delta_{j / M}
$$

to be the discrete probability measure giving equal weight to each point in the equidistributed set $\mathcal{P}_{M}$.

If $f:[0,1] \rightarrow \mathbb{R}$ is continuous, then its integral $\mu(f)$ with respect to Lebesgue measure $\mu$ is approximated by the sequence of values

$$
E_{M}(f)=\frac{1}{M+1} \sum_{j=0}^{M} f\left(\frac{j}{M}\right)
$$

as $M \rightarrow \infty$. Even if $f$ is rather regular, for example a polynomial, the rate at which the $E_{M}(f)$ approach $\mu(f)$ is typically no better than $O(1 / M)$ as $M \rightarrow \infty .^{1}$

The purpose of the present article is to show that this convergence can be significantly accelerated by considering linear combinations of the equidistributions $E_{M}$. More precisely, for any $n \in \mathbb{N}$ we shall consider the $n$ equidistributions

$$
\beta_{m}:=E_{2^{m}-1}, \quad 1 \leq m \leq n,
$$

and combine them so as to form a signed probability measure $\mu_{n}$.

Theorem 1. There is a sequence of signed probability measures $\mu_{n}$, each of which is a linear combination of the equidistributions $\beta_{m}, 1 \leq m \leq n$, such that for any real analytic function $f:[0,1] \rightarrow \mathbb{R}$, there exists $0<\theta<1$ such that

$$
\left|\mu(f)-\mu_{n}(f)\right|=O\left(\theta^{n^{2}}\right) \quad \text { as } n \rightarrow \infty .
$$

It is natural to relate the quality of the approximation to the number of evaluations of $f$ which need to be performed. Since each $\mathcal{P}_{n}$ has cardinality $2^{n}$, the signed measure $\mu_{n}$ has $N \leq 2^{n+1}$ atoms, and the error term is $O\left(\exp \left(-k(\log N)^{2}\right)\right)$ as $N \rightarrow \infty$, where the implied constant in this bound can be explicitly given. In particular, this error term is asymptotically smaller than $O\left(N^{-l}\right)$ for any $l>0$; thus, the convergence rate is better than for any Newton-Cotes integral rule.

The formulae for the $\mu_{n}$ are not particularly complicated. First, define the combinatorial coefficients

$$
e_{j}=(-1)^{j} \frac{2^{-j(j+1) / 2}}{\prod_{i=1}^{j}\left(1-2^{-i}\right)} .
$$

The first few combinatorial coefficients are

$$
e_{0}=1, \quad e_{1}=-1, \quad e_{2}=1 / 3, \quad e_{3}=-1 / 21, \quad e_{4}=1 / 315, \quad \text { etc. }
$$

Then define signed measures $\gamma_{n}$ by

$$
\gamma_{n}:=\sum_{m=1}^{n} \frac{e_{n-m}}{1-2^{-m}} \beta_{m} .
$$

The signed probability measures $\mu_{n}$ are by definition the normalised versions of the $\gamma_{n}$ :

$$
\mu_{n}:=\frac{\gamma_{n}}{\gamma_{n}(1)}=\left(\sum_{m=1}^{n} \frac{e_{n-m}}{1-2^{-m}}\right)^{-1} \gamma_{n} .
$$

\footnotetext{
${ }^{1}$ This bound is proved via the mean value theorem and is valid provided $f$ is $C^{1}$.
} 
For example, if $n=1$, then $\gamma_{1}=2 E_{1}$, so

$$
\mu_{1}=E_{1}=\frac{1}{2}\left(\delta_{0}+\delta_{1}\right) .
$$

If $n=2$, then $\gamma_{2}=-2 E_{1}+\frac{4}{3} E_{3}$, so

$$
\mu_{2}=3 E_{1}-2 E_{3}=\left(\delta_{0}+\delta_{1}\right)-\frac{1}{2}\left(\delta_{1 / 3}+\delta_{2 / 3}\right) .
$$

If $n=3$, then $\gamma_{3}=\frac{2}{3} E_{1}-\frac{4}{3} E_{3}+\frac{8}{7} E_{7}$, so

$$
\mu_{3}=\frac{7}{5} E_{1}-\frac{14}{5} E_{3}+\frac{12}{5} E_{7}=\frac{3}{10}\left(\delta_{0}+\delta_{1}\right)-\frac{7}{10}\left(\delta_{1 / 3}+\delta_{2 / 3}\right)+\frac{3}{10} \sum_{i=1}^{6} \delta_{i / 7} .
$$

Continuing in this way, each of the signed measures $\mu_{n}$ may be determined as in (6), (7), and (8), i.e., the weight associated to each of its atoms can be explicitly computed. To evaluate a given $\mu_{n}(f)$, it then only remains to evaluate $f$ at each of these atoms, though in practice it is usually easier to simply compute $\beta_{m}$ for $m=1, \ldots, n$ and then use the formula

$$
\mu_{n}(f)=\left(\sum_{m=1}^{n} \frac{e_{n-m}}{1-2^{-m}}\right)^{-1} \sum_{m=1}^{n} \frac{e_{n-m}}{1-2^{-m}} \beta_{m}(f) .
$$

Example 1. If $f(x)=\frac{5 \pi}{2}\left(e^{\pi}-2\right)^{-1} e^{\pi x} \cos (\pi x / 2)$, then $\mu(f)=1 .^{2}$ The first six approximations to $\mu(f)$ from equidistributions are

$$
\begin{array}{lll}
\beta_{1}(f)=0.185755, & \beta_{2}(f)=0.699195, & \beta_{3}(f)=0.876573, \\
\beta_{4}(f)=0.944025, & \beta_{5}(f)=0.973323, & \beta_{6}(f)=0.986974,
\end{array}
$$

while the corresponding approximations from the signed measures $\mu_{n}$ are

$$
\begin{array}{lll}
\mu_{1}(f)=0.185755, & \mu_{2}(f)=-0.841124, & \mu_{3}(f)=0.406088, \\
\mu_{4}(f)=1.092333, & \mu_{5}(f)=0.996815, & \mu_{6}(f)=1.000047 .
\end{array}
$$

When $n=10$, we have $\beta_{10}(f)=0.999204$ and

$$
\mu_{10}(f)=0.9999999999999936,
$$

while by the time $n=15$ we have $\beta_{15}(f)=0.999975$ and

$$
\mu_{15}(f)=0.999999999999999999999999999999999306 .
$$

By contrast, if Simpson's rule is used with the equidistributed sets $\mathcal{P}_{2^{n}-1}$, then the approximations to the integral exhibit slower convergence:

$$
\begin{array}{rr}
n=5: & 0.999999877708773828605590152248668712473 \\
n=10: & 0.999999999999883344326211018145038653466, \\
n=15: & 0.999999999999999999888748452411747628256 .
\end{array}
$$

\footnotetext{
${ }^{2}$ This example is from [13, p. 149], where an equivalent integral is used to illustrate an extrapolation method of integration.
} 
Remark 1. Each $\mathcal{P}_{2^{m}-1}$ is precisely the set of period- $m$ points for the $\times 2$-map $T:[0,1] \rightarrow[0,1]$ defined by $T(x)=2 x(\bmod 1)$ for $x \in[0,1)$, and $T(1)=1$. More generally, the $\times 2$-map could be replaced by the $\times q$-map (fixing the point 1 and mapping $x \in[0,1)$ to $q x(\bmod 1)$ ), for any integer $q \geq 3$, leading to different (though similar) formulae, and analogous results on the speed of approximation.

In order to prove Theorem 1, we first show, in Section 2, that the signed measures $\mu_{n}$ converge to Lebesgue measure $\mu$ in the weak-* topology. The error bound $\left|\mu(f)-\mu_{n}(f)\right|=O\left(\theta^{n^{2}}\right)$ for real analytic $f$ is then established in Section 3. In fact, a suitable modification of the definition of $\mu_{n}$ yields analogous results in any finite dimension $d$, as described in Section 4: if $f$ is real analytic, then $\left|\mu(f)-\mu_{n}(f)\right|=O\left(\theta^{n^{1+1 / d}}\right)$ as $n \rightarrow \infty$ (see Theorem 2).

\section{CONVERGENCE OF $\mu_{n}$ TO $\mu$}

Define

$$
\Delta(z)=\prod_{j=0}^{\infty}\left(1-\frac{z}{2^{j}}\right),
$$

an entire function of $z$ whose power series around 0 we write as

$$
\Delta(z)=\sum_{n=0}^{\infty} c_{n} z^{n}
$$

where clearly $c_{0}=1$. In fact, Euler's formula gives, for $n \geq 1$,

$$
c_{n}=(-1)^{n} \frac{2^{-n(n-1) / 2}}{\prod_{i=1}^{n}\left(1-2^{-i}\right)},
$$

and therefore (cf. (3))

$$
\sum_{j=0}^{n} c_{j}=(-1)^{n} \frac{2^{-n(n+1) / 2}}{\prod_{i=1}^{n}\left(1-2^{-i}\right)}=e_{n} .
$$

For any continuous function $f:[0,1] \rightarrow \mathbb{R}$ and $n \in \mathbb{N}$, define $a_{n, f}: \mathbb{C} \rightarrow \mathbb{C}$ by

$$
a_{n, f}(t)=\frac{\sum_{x \in \mathcal{P}_{2^{n}-1}} e^{t S_{n} f(x)}}{n\left(2^{n}-1\right)},
$$

where

$$
S_{n} f:=\sum_{i=0}^{n-1} f \circ T^{i} .
$$

For each $t \in \mathbb{C}$, the function

$$
z \mapsto \sum_{n=1}^{\infty} a_{n, f}(t) z^{n}
$$

is analytic inside its disc of convergence

$$
|z|<\left(\limsup _{n \rightarrow \infty}\left|a_{n, f}(t)\right|^{1 / n}\right)^{-1}=: r(t) .
$$

Therefore, the function $\Delta_{f}$ defined by

$$
\Delta_{f}(z, t):=\exp \left(-\sum_{n=1}^{\infty} a_{n, f}(t) z^{n}\right)
$$


is analytic in $\left\{(z, t) \in \mathbb{C}^{2}:|z|<r(t)\right\}$, and for fixed $t$ we write its power series around 0 as

$$
\Delta_{f}(z, t)=\sum_{n=0}^{\infty} c_{n, f}(t) z^{n}
$$

where clearly $c_{0, f}(t)=1$.

The case $t=0$ is of particular interest: here $a_{n, f}(0)=\frac{2^{n}}{n\left(2^{n}-1\right)}$, so $r(0)=1$, and moreover

$$
\sum_{n=1}^{\infty} a_{n, f}(0) z^{n}=\sum_{n=1}^{\infty} \frac{z^{n}}{n} \frac{2^{n}}{\left(2^{n}-1\right)}=\sum_{n=1}^{\infty} \frac{z^{n}}{n} \sum_{j=0}^{\infty} 2^{-n j}=\sum_{j=0}^{\infty} \sum_{n=1}^{\infty} \frac{\left(2^{-j} z\right)^{n}}{n}=-\sum_{j=0}^{\infty} \log \left(1-\frac{z}{2^{j}}\right),
$$

so

$$
\Delta_{f}(z, 0)=\exp \left(-\sum_{n=1}^{\infty} a_{n, f}(0) z^{n}\right)=\prod_{j=0}^{\infty}\left(1-\frac{z}{2^{j}}\right)=\Delta(z)
$$

hence, in particular,

$$
c_{n, f}(0)=c_{n} \quad \text { for all } n \in \mathbb{N} .
$$

The function (13) has a pole on the circle of radius $r(t)$ (at the point $r(t)$ if $t \in \mathbb{R}$ ), corresponding to a zero of $\Delta_{f}$. However, if the continuous function $f$ is sufficiently "regular," for example Lipschitz, then $\Delta_{f}(\cdot, t)$ can be analytically continued to a disc (centred at 0 ) of radius strictly larger than $r(t)$. This result, due to Ruelle [10], is proved by relating $\Delta_{f}(\cdot, t)$ to the transfer operator $\mathcal{L}_{t}$ defined by

$$
\mathcal{L}_{t} \varphi(x)=\frac{1}{2}\left(e^{t f(x / 2)} \varphi\left(\frac{x}{2}\right)+e^{t f((x+1) / 2)} \varphi\left(\frac{x+1}{2}\right)\right) .
$$

This operator preserves various Banach spaces. For example, if $f$ is Lipschitz, then $\mathcal{L}_{t}$ acts continuously on Lip, the space of all Lipschitz functions on $[0,1]$. If $f$ is real analytic (see Section 3), then, after suitable holomorphic extension to a complex domain $D$ containing $[0,1], \mathcal{L}_{t}$ is a continuous operator on $A^{2}(D)$, the Hilbert space of functions $\varphi$ holomorphic on $D$ such that $|\varphi|^{2}$ is integrable with respect to planar Lebesgue measure. In the latter case $\mathcal{L}_{t}$ is trace class, and it can be shown (see Lemma 2) that $n a_{n, f}(t)$ equals the $\operatorname{trace} \operatorname{tr}\left(\mathcal{L}_{t}^{n}\right)$ of $\mathcal{L}_{t}^{n}$; therefore,

$$
\Delta_{f}(z, t)=\exp \left(-\sum_{n=1}^{\infty} \frac{z^{n}}{n} \operatorname{tr}\left(\mathcal{L}_{t}^{n}\right)\right)=\operatorname{det}\left(I-z \mathcal{L}_{t}\right)
$$

the determinant of $\mathcal{L}_{t}$ (see, e.g., [11]), an entire function with the property that, for fixed $t$, its zeros are precisely the reciprocals of the eigenvalues of $\mathcal{L}_{t}$. For Lipschitz $f$, although $\mathcal{L}_{t}$ : Lip $\rightarrow \operatorname{Lip}$ is not trace class, or even compact, Ruelle [10] established the following properties of the "formal determinant" $\Delta_{f}$ :

Lemma 1. If $f:[0,1] \rightarrow \mathbb{R}$ is Lipschitz, then

(a) $\mathcal{L}_{t}$ : Lip $\rightarrow$ Lip has a simple isolated maximal eigenvalue $\lambda(t)$;

(b) for any fixed $t \in \mathbb{C}$, the function $z \mapsto \Delta_{f}(z, t)$ is analytic in a disc centred at 0 of radius strictly greater than $r(t)=|\lambda(t)|^{-1}$;

(c) $\Delta_{f}\left(\lambda(t)^{-1}, t\right)=0$ for all $t \in \mathbb{C}$.

Lemma 1 allows us to prove the following key step in the proof that $\mu_{n} \rightarrow \mu$ :

Proposition 1. For all Lipschitz functions $f:[0,1] \rightarrow \mathbb{R}$,

$$
\mu(f)=\frac{\partial_{t} \Delta_{f}(1,0)}{\Delta^{\prime}(1)}
$$


Proof. Perturbation theory of simple eigenvalues (see, e.g., [5]) implies that the function $t \mapsto \lambda(t)$ is analytic in a neighbourhood of 0 , with $\lambda(0)=r(0)^{-1}=1$, and that its derivative at 0 is given by

$$
\lambda^{\prime}(0)=\frac{l\left(\left(\left.\frac{d}{d t} \mathcal{L}_{t}\right|_{t=0}\right) v\right)}{l(v)},
$$

where $v$ denotes the eigenfunction of $\mathcal{L}_{0}$ and $l$ the eigenfunction of the dual $\mathcal{L}_{0}^{\prime}$ corresponding to the eigenvalue $\lambda(0)=1$. In our case $v$ is the constant function 1 and $l$ is Lebesgue measure $\mu$ (regarded as a linear functional), so $l(v)=1$. Moreover,

$$
\frac{d}{d t} \mathcal{L}_{t} v(x)=\frac{1}{2} \sum_{y \in T^{-1}(x)} f(y) e^{t f(y)} v(y)
$$

so

$$
\left(\left.\frac{d}{d t} \mathcal{L}_{t}\right|_{t=0}\right) v(x)=\frac{1}{2} \sum_{y \in T^{-1}(x)} f(y),
$$

so from (19)

$$
\lambda^{\prime}(0)=\int \frac{1}{2} \sum_{y \in T^{-1}(x)} f(y) d \mu(x)=\mu\left(\mathcal{L}_{0} f\right)=\mu(f) .
$$

Now $\Delta_{f}\left(\lambda(t)^{-1}, t\right)=0$ by Lemma $1(\mathrm{c})$, so implicit differentiation, which is legitimate by Lemma $1(\mathrm{~b})$, gives

$$
\lambda^{\prime}(0)=\frac{\partial_{t} \Delta_{f}\left(\lambda(0)^{-1}, 0\right)}{\partial_{z} \Delta_{f}\left(\lambda(0)^{-1}, 0\right)}=\frac{\partial_{t} \Delta_{f}(1,0)}{\partial_{z} \Delta_{f}(1,0)}=\frac{\partial_{t} \Delta_{f}(1,0)}{\Delta^{\prime}(0)}
$$

and combining with (20) gives (18).

Corollary 1. For all Lipschitz functions $f:[0,1] \rightarrow \mathbb{R}$,

$$
\mu(f)=\frac{\sum_{m=1}^{\infty} c_{m, f}^{\prime}(0)}{\sum_{m=1}^{\infty} m c_{m}} .
$$

Proof. Partially differentiate (14) with respect to each variable, then use (18).

Remark 2. The denominators in (18) and (21) are known explicitly: from the infinite product (9), we see that

$$
\Delta^{\prime}(1)=\sum_{m=1}^{\infty} m c_{m}=-\prod_{i=1}^{\infty}\left(1-2^{-i}\right) \approx-0.288788 .
$$

Corollary 1 implies that the Lebesgue integral $\mu(f)$ is approximated by the quotients $\sum_{m=1}^{N} c_{m, f}^{\prime}(0) / \sum_{m=1}^{N} m c_{m}$. The next result tells us that these quotients are precisely the integrals of $f$ with respect to the signed measures $\mu_{N}$ defined in Section 1.

Proposition 2. For all Lipschitz functions $f:[0,1] \rightarrow \mathbb{R}$ and all $N \in \mathbb{N}$,

$$
\mu_{N}(f)=\frac{\sum_{m=1}^{N} c_{m, f}^{\prime}(0)}{\sum_{m=1}^{N} m c_{m}} .
$$

Proof. Differentiating (14) and (15) with respect to $t$ and then setting $t=0$ gives

$$
\sum_{n=1}^{\infty} c_{n, f}^{\prime}(0) z^{n}=-\sum_{n=1}^{\infty} a_{n, f}^{\prime}(0) z^{n} \sum_{n=0}^{\infty} c_{n} z^{n},
$$


and truncating both sides of $(24)$ at the $N$ th power of $z$ gives

$$
\sum_{n=1}^{N} c_{n, f}^{\prime}(0) z^{n}=-\sum_{n=1}^{N} a_{n, f}^{\prime}(0) \sum_{i=0}^{N-n} c_{i} z^{i}
$$

Setting $z=1$ and then using (11) gives

$$
\sum_{n=1}^{N} c_{n, f}^{\prime}(0)=-\sum_{n=1}^{N} a_{n, f}^{\prime}(0) \sum_{i=0}^{N-n} c_{i}=-\sum_{n=1}^{N} a_{n, f}^{\prime}(0) e_{N-n} .
$$

Now

$$
a_{n, f}^{\prime}(0)=\frac{\beta_{n}(f)}{1-2^{-n}} \quad \text { for all } n \in \mathbb{N}
$$

because

$$
\sum_{x \in \mathcal{P}_{2^{n}}-1} S_{n} f(x)=n \sum_{x \in \mathcal{P}_{2^{n}-1}} f(x),
$$

so combining (4), (25), and (26) gives

$$
\sum_{n=1}^{N} c_{n, f}^{\prime}(0)=-\sum_{n=1}^{N} e_{N-n} \frac{\beta_{n}(f)}{1-2^{-n}}=-\gamma_{N}(f) .
$$

In view of (27) and the fact (cf. (5)) that $\mu_{N}(f):=\gamma_{N}(f) / \gamma_{N}(1)$, to prove (23) it remains to show that

$$
\gamma_{N}(1)=-\sum_{m=1}^{N} m c_{m}
$$

For this, first note that

$$
\gamma_{N}(1)=-\sum_{n=1}^{N} c_{n, 1}^{\prime}(0)
$$

by (27). Now

$$
a_{n, 1}(t)=\frac{e^{n t}}{n\left(1-2^{-n}\right)}
$$

so

$$
\Delta_{1}(z, t)=\Delta\left(z e^{t}\right)
$$

and therefore

$$
1+\sum_{m=1}^{\infty} c_{m, 1}(t) z^{m}=1+\sum_{m=1}^{\infty} c_{m} e^{t m} z^{m} .
$$

Comparing coefficients gives

$$
c_{m, 1}(t)=c_{m} e^{t m}
$$

for all $m \in \mathbb{N}$ and $t \in \mathbb{C}$, and differentiating at $t=0$ gives

$$
c_{m, 1}^{\prime}(0)=m c_{m} \quad \text { for all } m \in \mathbb{N} .
$$

Substituting (30) into (29) gives (28), as required.

Combining Corollary 1 and Proposition 2 gives

Corollary 2. For all Lipschitz functions $f:[0,1] \rightarrow \mathbb{R}$,

$$
\mu_{n}(f) \rightarrow \mu(f) \quad \text { as } n \rightarrow \infty \text {. }
$$


Since Lipschitz functions are uniformly dense in the space of continuous functions, we deduce

Corollary 3. The sequence of signed measures $\mu_{n}$ converges, in the weak-* sense, to Lebesgue measure $\mu$.

Remark 3. In the sequel we shall mainly focus on real analytic functions $f:[0,1] \rightarrow \mathbb{R}$. Since such functions are also uniformly dense in the space of continuous functions, we could have established Corollary 3 by appealing to the (more classical) interpretation of $\Delta_{f}$ as a bona fide determinant, rather than Ruelle's Lemma 1. However, the intervening results of this section may be of independent interest, and it seemed appropriate to indicate their range of validity. In fact, the Lipschitz condition can be weakened, e.g., to Hölder continuity or, more generally, to any $f$ for which an analogue of Lemma 1 can be established for $\mathcal{L}_{t}$ and $\Delta_{f}$.

\section{SPEED OF CONVERGENCE OF $\mu_{n}(f)$}

In this section we will prove Theorem 1 , that if $f:[0,1] \rightarrow \mathbb{R}$ is real analytic, then the $\mu_{n}(f)$ approximate $\mu(f)$ at rate $O\left(\theta^{n^{2}}\right)$ for some $0<\theta<1$.

The function $f:[0,1] \rightarrow \mathbb{R}$ has a holomorphic extension to some simply connected complex domain $D$ containing $[0,1]$. Clearly $D$ may be chosen so as to contain the closure of $T_{0}(D) \cup T_{1}(D)$, where $T_{0}(z):=z / 2$ and $T_{1}(z):=(z+1) / 2$. Let $A^{2}(D)$ be the Bergman space consisting of functions $\varphi$ holomorphic on $D$ such that $|\varphi|^{2}$ is integrable with respect to planar Lebesgue measure $A$. This is a Hilbert space when equipped with the inner product $(\varphi, \psi)=\int_{D} \varphi \bar{\psi} d A$. Formula (17) defines a bounded linear operator $\mathcal{L}_{t}: A^{2}(D) \rightarrow A^{2}(D)$. We will show that the approximation numbers

$$
a_{n}\left(\mathcal{L}_{t}\right)=\inf \left\{\left\|\mathcal{L}_{t}-L\right\|: L \text { is linear with } \operatorname{rank}(L)<n\right\}
$$

decrease exponentially with $n$ (cf. similar arguments in, e.g., $[1,4]$ ).

For simplicity suppose that $D$ may be chosen to be an open Euclidean disc (this is possible for a dense set of $f$, for example if $f$ is a polynomial; more generally, we may transform the simply connected domain $D$ to a disc via a Riemann mapping). Let $D^{\prime \prime}$ be a smaller open disc, concentric with $D$ and containing $T_{0}(D) \cup T_{1}(D)$, and let $H^{\infty}\left(D^{\prime \prime}\right)$ denote the set of bounded holomorphic functions on $D^{\prime \prime}$, a Banach space when equipped with the supremum norm. Let $D^{\prime}$ be another concentric open disc, strictly smaller than $D$ and strictly larger than $D^{\prime \prime}$. There are natural embeddings $J: A^{2}(D) \hookrightarrow A^{2}\left(D^{\prime}\right)$ and $J^{\prime}: A^{2}\left(D^{\prime}\right) \hookrightarrow H^{\infty}\left(D^{\prime \prime}\right)$, defined by simply restricting the domain of definition. Since $T_{0}(D) \cup T_{1}(D) \subset D^{\prime \prime}$, if $\varphi \in H^{\infty}\left(D^{\prime \prime}\right)$ then $\mathcal{L}_{t} \varphi \in A^{2}(D)$, so $\mathcal{L}_{t}$ extends to an operator $\widetilde{\mathcal{L}}_{t}: H^{\infty}\left(D^{\prime \prime}\right) \rightarrow A^{2}(D)$. A short calculation shows this operator to be bounded, with

$$
\left\|\widetilde{\mathcal{L}}_{t}\right\| \leq \sup _{z \in D}\left(\left|e^{t f(z / 2)}\right|+\left|e^{t f((z+1) / 2)}\right|\right) .
$$

Now $J$ and $J^{\prime}$ are bounded, and since $\mathcal{L}_{t}=\widetilde{\mathcal{L}}_{t} J^{\prime} J$, we have $a_{n}\left(\mathcal{L}_{t}\right) \leq\left\|\widetilde{\mathcal{L}}_{t}\right\| \cdot\left\|J^{\prime}\right\| a_{n}(J)$ for all $n \geq 1$ (see [8, 2.2]). But the $a_{n}(J)$ are known exactly (see, e.g., [9]): if $r<1$ denotes the ratio of the radii of $D^{\prime}$ and $D$, then $a_{n}(J)=r^{n-1}$. Therefore,

$$
a_{n}\left(\mathcal{L}_{t}\right) \leq\left\|\widetilde{\mathcal{L}_{t}}\right\| \cdot\left\|J^{\prime}\right\| r^{n-1} \quad \text { for all } n \geq 1 .
$$

Now, as mentioned briefly in Section 2, the function $z \mapsto \Delta_{f}(z, t)$ is the determinant $\operatorname{det}\left(I-z \mathcal{L}_{t}\right)$ of $\mathcal{L}_{t}$, defined for $|z|$ small by

$$
\operatorname{det}\left(I-z \mathcal{L}_{t}\right)=\exp \left[\operatorname{tr}\left(\log \left(I-z \mathcal{L}_{t}\right)\right)\right]=\exp \left(-\sum_{n=1}^{\infty} \frac{z^{n}}{n} \operatorname{tr}\left(\mathcal{L}_{t}^{n}\right)\right),
$$

and with a holomorphic extension to the entire $z$-plane (see, e.g., [11, Theorem 3.3]), a standard result (see, e.g., [7]) whose proof we sketch below. 
Lemma 2.

$$
\Delta_{f}(z, t)=\operatorname{det}\left(I-z \mathcal{L}_{t}\right)
$$

Proof. Write $\underline{i}=\left(i_{1}, \ldots, i_{n}\right)$ and $T_{\underline{i}}=T_{i_{n}} \circ \ldots \circ T_{i_{1}}$, so that

$$
\mathcal{L}_{t}^{n}=\sum_{\underline{i} \in\{0,1\}^{n}} \mathcal{L}_{\underline{i}}
$$

where

$$
\mathcal{L}_{\underline{i}} \varphi(z):=2^{-n} e^{t S_{n} f\left(T_{\underline{i}} z\right)} \varphi\left(T_{\underline{i}} z\right) .
$$

Now each $\mathcal{L}_{\underline{i}}: A^{2}(D) \rightarrow A^{2}(D)$ is a weighted composition operator, and as such (see [2]) its eigenvalues (all simple) are the values

$$
2^{-n} e^{t S_{n} f\left(z_{i}\right)} T_{\underline{i}}^{\prime}\left(z_{i}\right)^{k}, \quad k=0,1, \ldots, \infty,
$$

where $z_{\underline{i}}$ denotes the unique fixed point of $T_{\underline{i}}$. Now $T_{\underline{\underline{i}}}^{\prime}\left(z_{\underline{i}}\right)=2^{-n}$, so

$$
\operatorname{tr}\left(\mathcal{L}_{\underline{i}}\right)=\frac{e^{t S_{n} f\left(z_{\underline{i}}\right)}}{2^{n}-1}
$$

and the set $\left\{z_{\underline{i}}: \underline{i} \in\{0,1\}^{n}\right\}$ is precisely $\mathcal{P}_{2^{n}-1}$; so combining (35) and (36) gives

$$
\operatorname{tr}\left(\mathcal{L}_{t}^{n}\right)=\sum_{x \in \mathcal{P}_{2^{n}}-1} \frac{e^{t S_{n} f^{n}(x)}}{2^{n}-1}=n a_{n, f}(t),
$$

and comparing (14) and (33) yields (34), as required.

Lemma 3. There exist $0<s<1$ and $C>0$ such that

$$
\left|c_{m, f}^{\prime}(0)\right| \leq C s^{m(m+1) / 2} \quad \text { for all } m \geq 1 .
$$

Proof. Let $\left\{\lambda_{k}\left(\mathcal{L}_{t}\right)\right\}_{k=1}^{\infty}$ denote the eigenvalue sequence of $\mathcal{L}_{t}$ (i.e., eigenvalues listed by nondecreasing modulus and according to algebraic multiplicity). By [11, Lemma 3.3], the Taylor coefficients $c_{m, f}(t)$ of $\Delta_{f}(z, t)=\operatorname{det}\left(I-z \mathcal{L}_{t}\right)$ are given by $c_{m, f}(t)=\sum_{i_{1}<\ldots<i_{m}} \lambda_{i_{1}}\left(\mathcal{L}_{t}\right) \ldots \lambda_{i_{m}}\left(\mathcal{L}_{t}\right)$, the summation being over all $m$-tuples $\left(i_{1}, \ldots, i_{m}\right)$ of positive integers satisfying $i_{1}<\ldots<i_{m}$. Thus,

$$
\begin{aligned}
\left|c_{m, f}(t)\right| & =\left|\sum_{i_{1}<\ldots<i_{m}} \lambda_{i_{1}}\left(\mathcal{L}_{t}\right) \ldots \lambda_{i_{m}}\left(\mathcal{L}_{t}\right)\right| \leq \sum_{i_{1}<\ldots<i_{m}} a_{i_{1}}\left(\mathcal{L}_{t}\right) \ldots a_{i_{m}}\left(\mathcal{L}_{t}\right) \\
& \leq\left(\frac{\left\|\widetilde{\mathcal{L}}_{t}\right\| \cdot\left\|J^{\prime}\right\|}{r}\right)^{m} \sum_{i_{1}<\ldots<i_{m}} r^{i_{1}+\ldots+i_{m}}=\left(\frac{\left\|\widetilde{\mathcal{L}}_{t}\right\| \cdot\left\|J^{\prime}\right\|}{r}\right)^{m} \frac{r^{m(m+1) / 2}}{(1-r)\left(1-r^{2}\right) \ldots\left(1-r^{m}\right)} \\
& \leq b(r) \frac{\left\|J^{\prime}\right\|^{m}}{r^{m}}\left(\sup _{z \in D}\left(\left|e^{t f(z / 2)}\right|+\left|e^{t f((z+1) / 2)}\right|\right)\right)^{m} r^{m(m+1) / 2},
\end{aligned}
$$

where $b(r):=\prod_{i=1}^{\infty}\left(1-r^{i}\right)^{-1}<\infty$. The first inequality in the above calculation is by [3, Corollary VI.2.6], the second by (32), the next step is an $m$-fold geometric summation, and the final step uses (31).

Fix $\varepsilon>0$, and let $K_{1}>0$ be such that

$$
\sup _{|t| \leq \varepsilon} \sup _{z \in D}\left(\left|e^{t f(z / 2)}\right|+\left|e^{t f((z+1) / 2)}\right|\right)<K_{1} .
$$


So $\left|c_{m, f}(t)\right| \leq b(r) \frac{\left\|J^{\prime}\right\|}{r^{m}} K_{1}^{m} r^{m(m+1) / 2}$ for all $m \geq 1$; thus, for any $s \in(r, 1)$ there exists $K_{2}>0$ such that

$$
\left|c_{m, f}(t)\right| \leq K_{2} s^{m(m+1) / 2} \quad \text { for all } m \geq 1 .
$$

If $\Gamma$ denotes the positively oriented circle of radius $\varepsilon$ centred at 0 , then Cauchy's integral formula combined with (38) gives, for all $m \geq 1$,

$$
\left|c_{m, f}^{\prime}(0)\right|=\left|\frac{1}{2 \pi i} \int_{\Gamma} \frac{c_{m, f}(t)}{t^{2}} d t\right| \leq \varepsilon^{-2} K_{2} s^{m(m+1) / 2},
$$

as required.

To conclude the proof of Theorem 1, first note that if $0<\alpha<1$, then

$$
\sum_{n=N}^{\infty} \alpha^{n^{2}} \leq \sum_{n=N}^{\infty} \alpha^{n N}=\frac{\alpha^{N^{2}}}{1-\alpha^{N}}=O\left(\alpha^{N^{2}}\right) \quad \text { as } \quad N \rightarrow \infty .
$$

It therefore follows from (10) that

$$
A_{N}:=\left|\sum_{n=N+1}^{\infty} n c_{n}\right|=O\left(\alpha^{N^{2}}\right) \quad \text { as } N \rightarrow \infty
$$

for any $1 / \sqrt{2}<\alpha<1$, and from Lemma 3 that

$$
B_{N}:=\left|\sum_{n=N+1}^{\infty} c_{n, f}^{\prime}(0)\right|=O\left(\beta^{N^{2}}\right) \quad \text { as } N \rightarrow \infty
$$

for some $0<\beta<1$ (in fact, $\sqrt{s}<\beta<1$ with $s$ as in Lemma 3).

Now $\left|\Delta^{\prime}(1)\right| \cdot\left|\sum_{n=1}^{N} n c_{n}\right| \rightarrow \Delta^{\prime}(1)^{2} \approx 0.0834 \neq 0$ as $N \rightarrow \infty$, so (39) and (40) imply that

$$
\left|\mu(f)-\mu_{N}(f)\right| \leq \frac{\left|\partial_{t} \Delta_{f}(1,0)\right| A_{N}+\left|\Delta^{\prime}(1)\right| B_{N}}{\left|\Delta^{\prime}(1)\right| \cdot\left|\sum_{n=1}^{N} n c_{n}\right|}=O\left(\theta^{N^{2}}\right) \quad \text { as } \quad N \rightarrow \infty,
$$

where

$$
\theta=\max (\alpha, \beta) \in\left(\frac{1}{\sqrt{2}}, 1\right)
$$

Remark 4. We noted above that $\alpha$ can be chosen arbitrarily in the range $1 / \sqrt{2}<\alpha<1$. If $f:[0,1] \rightarrow \mathbb{R}$ extends to an entire function (e.g., if it is a polynomial), then $\beta$ may also be chosen arbitrarily in the range $1 / \sqrt{2}<\beta<1$. This is because the discs $D$ may be chosen arbitrarily large, and since $T_{0}$ and $T_{1}$ contract distances by $1 / 2$, the value $r$, and hence $s$, may be chosen arbitrarily close to $1 / 2$. Therefore, the value $\theta=\max (\alpha, \beta)$ in Theorem 1 can be chosen arbitrarily close to $1 / \sqrt{2}$.

Remark 5. A natural question is whether the methods of this paper generalise to the circle $\mathbb{R} / \mathbb{Z}$, or more generally to the $d$-dimensional torus (cf. Section 4 ). In other words, can numerical integration of analytic functions using equidistributed points be accelerated in the same way as on the interval?

The answer is no: it turns out that the signed measures $\mu_{n}$ are in this case precisely the equidistributions $\beta_{n}=E_{2^{n}-1}$, giving weight $1 /\left(2^{n}-1\right)$ to each point in the set $\mathcal{P}_{2^{n}-1}:=\left\{j /\left(2^{n}-1\right)\right.$ : $\left.0 \leq j \leq 2^{n}-2\right\}$. On the other hand, the approximations $\beta_{n}(f)$, or more generally $E_{p}(f)$, to the Lebesgue integral $\mu(f)$ are significantly faster than on the interval, as we now explain. 
If $a_{n, f}(t)$ is defined as in (12) and $\Delta_{f}$ as in (14), then, because $\mathcal{P}_{2^{n}-1}$ has cardinality $2^{n}-1$,

$$
\Delta_{f}(z, 0)=\exp \left(-\sum_{n=1}^{\infty} \frac{z^{n}}{n} \frac{\# \mathcal{P}_{2^{n}-1}}{2^{n}-1}\right)=\exp \log (1-z)=1-z,
$$

so the function $\Delta(z):=1-z$ replaces the definition of $\Delta$ in (9). In particular, the Taylor coefficients $c_{n}$ of $\Delta$ are simply $c_{0}=1, c_{1}=-1$, and $c_{n}=0$ for $n \geq 2$, so if $e_{n}:=\sum_{i=0}^{n} c_{i}$, then $e_{0}=1$ and $e_{n}=0$ for $n \geq 1$.

Therefore, $\gamma_{n}:=\sum_{m=1}^{n} \frac{e_{n-m}}{1-2^{-m}} \beta_{m}=\frac{\beta_{n}}{1-2^{-n}}$, and its normalisation $\mu_{n}$ is precisely the equidistribution $\beta_{n}{ }^{3}$

Now we claim ${ }^{4}$ that, for real analytic functions $f$ on the circle (which now we represent as $S^{1}=$ $\{z \in \mathbb{C}:|z|=1\})$, the error $\left|\mu(f)-E_{p}(f)\right|$ is $O\left(\gamma^{p}\right)$ as $p \rightarrow \infty$ for some $0<\gamma<1$; in particular,

$$
\left|\mu(f)-\beta_{n}(f)\right|=\left|\mu(f)-\mu_{n}(f)\right|=O\left(\gamma^{2^{n}}\right) \quad \text { as } n \rightarrow \infty .
$$

The equidistributed set $\mathcal{P}_{p}$ is, in multiplicative notation, the set $U_{p}$ of $p$ th roots of unity. Analyticity of $f$ means that its Fourier coefficients $f_{n}:=\int f(z) z^{-n} d \mu(z)$ satisfy $\left|f_{n}\right| \leq C \gamma^{n}$ for all $n \geq 1$ and some $0<\gamma<1$ and $C>0$. Moreover, $\mu(f)=f_{0}$. Writing $U_{p}=\left\{z_{1}, \ldots, z_{p}\right\}$, we have

$$
\sum_{z \in U_{p}} f(z)=\sum_{j=1}^{p} \sum_{n=0}^{\infty} f_{n} z_{j}^{n}=\sum_{n=0}^{\infty} f_{n} \sum_{j=1}^{p} z_{j}^{n}
$$

but

$$
\sum_{j=1}^{p} z_{j}^{n}= \begin{cases}p & \text { if } p \mid n \\ 0 & \text { otherwise }\end{cases}
$$

so $E_{p}(f)=\frac{1}{p} \sum_{z \in U_{p}} f(z)=\sum_{n=0}^{\infty} f_{n p}$, and therefore

$$
\left|\mu(f)-E_{p}(f)\right|=\left|\sum_{n=1}^{\infty} f_{n p}\right| \leq \sum_{n=1}^{\infty}\left|f_{n p}\right| \leq C \sum_{n=1}^{\infty} \gamma^{n p}=O\left(\gamma^{p}\right) \quad \text { as } \quad p \rightarrow \infty .
$$

\section{MULTIDIMENSIONAL INTEGRALS}

All of the results described so far have higher dimensional analogues. Let $\mu$ denote $d$-dimensional Lebesgue measure. We shall describe these results in the case of the unit cube $[0,1]^{d}$, though minor modifications allow numerical integration over any self-similar domain $X \subset \mathbb{R}^{d}$, i.e. one which can be written as $X=\sum_{i=1}^{2^{d}} T_{i}(X)$, where each $T_{i}$ is an affine map with Jacobian $2^{-d}$.

Define the equidistributed set

$$
\mathcal{P}_{M}=\left\{\left(\frac{j_{1}}{M}, \ldots, \frac{j_{d}}{M}\right):\left(j_{1}, \ldots, j_{d}\right) \in\{0,1, \ldots, M\}^{d}\right\}
$$

the equidistribution

$$
E_{M}=\frac{1}{(M+1)^{d}} \sum_{x \in \mathcal{P}_{M}} \delta_{x}
$$

and again set

$$
\beta_{m}=E_{2^{m}-1} .
$$

\footnotetext{
${ }^{3}$ An almost identical computation shows that $\mu_{n}=\beta_{n}$ on the $d$-dimensional torus as well, cf. Section 4 .

${ }^{4}$ Undoubtedly this argument is not new, though we are not aware of a precise reference.
} 
The support $\mathcal{P}_{2^{m}-1}$ of $\beta_{m}$ is precisely the set of period- $m$ points for the map $T:=T_{1} \times \ldots \times T_{1}$ : $[0,1]^{d} \rightarrow[0,1]^{d}$, where we now abuse notation by using $T_{1}$ to denote the map formerly known as $T$.

The $d$-dimensional analogue of Theorem 1 is

Theorem 2. There is a sequence of signed probability measures $\mu_{n}$, each of which is a linear combination of the equidistributions $\beta_{m}, 1 \leq m \leq n$, such that for any real analytic function $f:[0,1]^{d} \rightarrow \mathbb{R}$, there exists $0<\theta<1$ such that

$$
\left|\mu(f)-\mu_{n}(f)\right|=O\left(\theta^{n^{1+1 / d}}\right) \quad \text { as } n \rightarrow \infty .
$$

In other words, since each $\mathcal{P}_{n}$ has cardinality $2^{d n}$, the signed measure $\mu_{n}$ has $N \leq 2^{d(n+1)}$ atoms, and the error term is $O\left(\exp \left(-k(\log N)^{1+1 / d}\right)\right)$ as $N \rightarrow \infty$.

In dimension $d>1$, the combinatorial coefficients $e_{n}$ are defined implicitly in terms of the entire function $^{5}$

$$
\Delta(z)=\prod_{j=0}^{\infty}\left(1-\frac{z}{2^{j}}\right)^{\left(\begin{array}{c}
d+j-1 \\
j
\end{array}\right)},
$$

where $\left(\begin{array}{c}d+j-1 \\ j\end{array}\right)=\frac{(d+j-1) !}{(d-1) ! j !}$, whose power series we again write ${ }^{6}$ as

$$
\Delta(z)=\sum_{n=0}^{\infty} c_{n} z^{n}
$$

with $c_{0}=1$. We then define

$$
e_{n}=\sum_{j=0}^{n} c_{n}
$$

Remark 6. The definition (42) is an analogue of (3). The difference is that when $d=1$ we have a closed-form expression for $e_{n}$ and $c_{n}$ (cf. (3) and (10)), whereas for $d>1$ we do not. Nevertheless, these coefficients may be generated recursively and tabulated (see below).

The signed measures $\gamma_{n}$ are defined by

$$
\gamma_{n}:=\sum_{m=1}^{n} \frac{e_{n-m}}{\left(1-2^{-m}\right)^{d}} \beta_{m},
$$

and again the $\mu_{n}$ are just their normalised versions:

$$
\mu_{n}:=\left(\sum_{m=1}^{n} \frac{e_{n-m}}{\left(1-2^{-m}\right)^{d}}\right)^{-1} \gamma_{n} .
$$

Remark 7. We may derive an explicit formula for the weights corresponding to each atom of $\mu_{n}$. If $w_{x, n}$ denotes the weight attached to the atom $x$ of $\mu_{n}$, then

$$
w_{x, n}=\frac{\sum_{\substack{1 \leq m \leq n \\ \operatorname{per}(x) \mid m}} \frac{e_{n-m}}{\left(2^{m}-1\right)^{d}}}{\sum_{1 \leq m \leq n} \frac{e_{n-m}}{\left(1-2^{-m}\right)^{d}}},
$$

where $\operatorname{per}(x)$ is the least period of $x$ (i.e., the smallest natural number $k$ such that $T^{k}(x)=x$ ).

\footnotetext{
${ }^{5}$ Note that when $d=1$, the function $\Delta$ is as in (9).

${ }^{6}$ Throughout this section we suppress the dependence on the dimension $d$ from the notation, this being obvious from the context.
} 
The proof of Theorem 2 is similar to that of Theorem 1, so here we simply sketch the main differences. Setting

$$
a_{n, f}(t)=\frac{\sum_{x \in \mathcal{P}_{2^{n}-1}} e^{t S_{n} f(x)}}{n\left(2^{n}-1\right)^{d}},
$$

we define the function $\Delta_{f}$ by the formula (14), an analytic function of $z$ whose power series around 0 we again write as $\Delta_{f}(z, t)=\sum_{n=0}^{\infty} c_{n, f}(t) z^{n}$. Again $\Delta_{f}(z, 0)=\Delta(z)$, so that $c_{n, f}(0)=c_{n}$ for all $n \geq 0$. The results from Section 2 are then true verbatim, and the proofs are identical; in particular, $\mu_{n} \rightarrow \mu$ in the weak-* topology. The only difference is that in general $\Delta^{\prime}(1)=-\prod_{i=1}^{\infty}\left(1-2^{-i}\right)\left({ }_{i}^{d+i-1}\right)$ (rather than the value in (22)).

To establish the speed of convergence $O\left(\theta^{n^{1+1 / d}}\right)$ for real analytic $f$, we first show that $z \mapsto$ $\Delta_{f}(z, t)$ is the determinant of $\mathcal{L}_{t}: A^{2}(D) \rightarrow A^{2}(D)$ (with $D$ a domain mapped compactly inside itself by the inverse branches of $T$, and $A^{2}(D)$ the corresponding Bergman space defined in terms of $2 d$-dimensional Lebesgue measure); here the principal difference in proving the trace formulae (cf. Lemma 2) is that for $d>1$ the eigenvalues of the weighted composition operators are no longer simple (see, e.g., [2] for further details). Another significant difference is that the approximation numbers $a_{n}\left(\mathcal{L}_{t}\right)$ are $O\left(r^{n^{1 / d}}\right)$ as $n \rightarrow \infty$, for some $0<r<1$. However, unlike for $d=1$, we cannot use a Riemann mapping to reduce to the case where $D$ has a simple geometry; the proof of the asymptotic $a_{n}\left(\mathcal{L}_{t}\right)=O\left(r^{n^{1 / d}}\right)$ for general domains $D$ is elaborated in [1]. With this stretched exponential estimate on the approximation numbers of $\mathcal{L}_{t}$, we derive the bound $O\left(\theta^{n^{1+1 / d}}\right)$ on the coefficients $n c_{n}$ and $c_{n, f}^{\prime}(0)$ as in Lemma 3, and hence an analogous bound on the error terms $\left|\mu(f)-\mu_{n}(f)\right|$.

Now we make explicit, in dimension $d=2$, the combinatorial coefficients $e_{n}$ defined by (42). We adopt the following temporary notation: let $\Delta^{(1)}$ and $\Delta^{(2)}$ denote, respectively, the functions $\Delta$ defined by (9) and (41), and write $\Delta^{(i)}(z)=\sum_{n=1}^{\infty} c_{i, n} z^{n}$ for $i=1,2$. The factorisation

$$
\Delta^{(2)}(z)=\Delta^{(1)}(z) \Delta^{(2)}(z / 2)
$$

yields the recurrence relation

$$
c_{2, n}=\frac{1}{1-2^{-n}} \sum_{m=0}^{n-1} 2^{-m} c_{2, m} c_{1, n-m},
$$

from which we can calculate the first few coefficients $c_{2, n}$, and hence the corresponding 2-dimensional combinatorial coefficients $e_{n}=\sum_{j=0}^{n} c_{2, n}$, as follows:

$$
\begin{aligned}
e_{0} & =1, & e_{1} & =-3, \\
e_{2} & =\frac{37}{9}=4.111111111, & e_{3} & =-\frac{505}{147}=-3.435374150, \\
e_{4} & =\frac{195701}{99225}=1.972295288, & e_{5} & =-\frac{26417561}{31785075}=-0.8311309947, \\
e_{6} & =0.2682138472, & e_{7} & =-0.06828271081, \\
e_{8} & =0.01402036233, & e_{9} & =-0.002362036477, \\
e_{10} & =0.0003310411037, & e_{11} & =-0.000039037128316283188611, \\
e_{12} & =0.39103988780032495342 \times 10^{-5}, & e_{13} & =-0.33547198246660495047 \times 10^{-6}, \\
e_{14} & =0.24823109138064728029 \times 10^{-7}, & e_{15} & =-0.15941012050087237023 \times 10^{-8},
\end{aligned}
$$




$$
\begin{aligned}
& e_{16}=0.89335294322654910042 \times 10^{-10}, \\
& e_{17}=-0.439047477554514229382481419383 \times 10^{-11}, \\
& e_{18}=0.190065123059235610472306457018 \times 10^{-12}, \\
& e_{19}=-0.727674920276166018169601798505 \times 10^{-14}, \\
& e_{20}=0.247288673429082442638851033326 \times 10^{-15} .
\end{aligned}
$$

Example 2. If $f:[0,1]^{2} \rightarrow \mathbb{R}$ is defined by $f(x, y)=x^{2}(x+y)$, then

$$
\mu(f)=\int_{0}^{1} \int_{0}^{1} f(x, y) d x d y=\frac{5}{12}=0.41666666666 \ldots \ldots
$$

Some of the uniform approximations $\beta_{n}(f)$ are

$$
\begin{aligned}
n=5: & \frac{53}{124}=0.4274193548, & n=10: & \frac{5119}{12276}=0.4169925057, \\
n=15: & \frac{54613}{131068}=0.4166768395, & n=20: & \frac{5242879}{12582900}=0.4166669845,
\end{aligned}
$$

while some of the approximations $\mu_{n}(f)$ are

$$
\begin{array}{llll}
n=5: & \frac{1286211440413}{1490974480380}=0.862664, & n=10: & 0.338034, \\
n=15: & 0.4166672262176816, & n=20: & 0.41666666666665389 .
\end{array}
$$

Remark 8. Since every compact smooth manifold admits a triangulation (see, e.g., [12, p. 579]), i.e. a decomposition into simplices, the methods of this article can in principle be used to perform numerical integration on manifolds.

In the case of the standard 2-sphere, a deep result of Lubotzky, Phillips, and Sarnak [6] uses Deligne's solution of the Ramanujan conjecture to construct sets $\mathcal{P}$ of "equidistributed" points on the 2 -sphere, arising from the orbit of an initial point under a suitable group. The discrete probability measure giving equal weight to each point in $\mathcal{P}$ is an approximation to Lebesgue measure $\mu$ on the sphere, and the error term in the approximation of a given integral $\mu(f)$ is shown to be $O\left(\frac{\log N}{N^{1 / 2}}\right)$ as the cardinality $N$ of $\mathcal{P}$ tends to infinity.

The methods of this paper can be used to give a rapid numerical integration algorithm on the 2 -sphere $S^{2}$; the idea is to consider an inscribed cube inside $S^{2}$ and then project outwards. If the faces of the cube are parallel to the coordinate axes, then the eight vertices are at $( \pm 1 / \sqrt{3}, \pm 1 / \sqrt{3}, \pm 1 / \sqrt{3})$, and if $F$ denotes the "bottom" face of the cube, in the plane $z=-1 / \sqrt{3}$, then the projection $p: F \rightarrow S^{2}$ is given by

$$
p(x, y,-1 / \sqrt{3})=\frac{(x, y,-1 / \sqrt{3})}{\sqrt{x^{2}+y^{2}+1 / 3}} .
$$

The integral of $f: S^{2} \rightarrow \mathbb{R}$ over the curvilinear square region $p(F)$ can be transformed into an integral over the face $F$,

$$
\int_{p(F)} f d \mu_{S^{2}}=\int_{F} f \circ p \operatorname{Jac}(p) d \mu_{F}=\int_{F} f\left(\frac{(x, y,-\sqrt{3})}{\sqrt{x^{2}+y^{2}+1 / 3}}\right) \frac{d x d y}{3\left(x^{2}+y^{2}+1 / 3\right)^{2}},
$$


and $F$ can be mapped linearly onto the unit cube $[0,1]^{2} \subset \mathbb{R}^{2}$, in order to use the formulae of Section 4. If $f: S^{2} \rightarrow \mathbb{R}$ is real analytic, then the error term in approximating $\mu(f)$ is $O\left(\exp \left(-k(\log N)^{3 / 2}\right)\right)$ as the cardinality $N$ of the support of the discrete signed measures tends to infinity.

\section{REFERENCES}

1. O. Bandtlow and O. Jenkinson, "Explicit Eigenvalue Estimates for Transfer Operators Acting on Spaces of Holomorphic Functions" (in preparation).

2. C. C. Cowen and B. D. MacCluer, Composition Operators on Spaces of Analytic Functions (CRC Press, Boca Raton, FL, 1995).

3. I. Gohberg, S. Goldberg, and M. A. Kaashoek, Classes of Linear Operators (Birkhäuser, Basel, 1990), Vol. 1.

4. O. Jenkinson and M. Pollicott, "Orthonormal Expansions of Invariant Densities for Expanding Maps," Adv. Math. 192, 1-34 (2005).

5. T. Kato, Perturbation Theory for Linear Operators (Springer, Berlin, 1966), Grundl. Math. Wiss. 132.

6. A. Lubotzky, R. Phillips, and P. Sarnak, "Hecke Operators and Distributing Points on the Sphere. I," Commun. Pure Appl. Math. 39, Suppl., S149-S186 (1986).

7. D. H. Mayer, "On a $\zeta$ Function Related to the Continued Fraction Transformation," Bull. Soc. Math. France 104, 195-203 (1976).

8. A. Pietsch, Eigenvalues and s-Numbers (Cambridge Univ. Press, Cambridge, 1987).

9. A. Pinkus, $n$-Widths in Approximation Theory (Springer, Berlin, 1985).

10. D. Ruelle, "An Extension of the Theory of Fredholm Determinants," Publ. Math., Inst. Hautes Étud. Sci. 72, 175-193 (1990).

11. B. Simon, Trace Ideals and Their Applications (Cambridge Univ. Press, Cambridge, 1979), LMS Lect. Note Ser. 35.

12. M. Spivak, A Comprehensive Introduction to Differential Geometry, 2nd ed. (Publish or Perish, Berkeley, 1979), Vol. 1.

13. J. Stoer and R. Bulirsch, Introduction to Numerical Analysis, 2nd ed. (Springer, New York, 1993).

This article was submitted by the authors in English 